\title{
A characteristic MRI finding to diagnose a partial tear of the medial meniscus posterior root: an ocarina sign
}

Takayuki Furumatsu* (0), Takaaki Hiranaka, Keisuke Kintaka, Yuki Okazaki, Naohiro Higashihara, Masanori Tamura and Toshifumi Ozaki

\begin{abstract}
Background: Diagnosing partial tears of the medial meniscus (MM) posterior root is difficult. The aim of this study was to evaluate diagnostic values involved in conventional magnetic resonance imaging (MRI) features of MM posterior root tears (MMPRTs) and find other MRI-based findings in patients with partial MMPRTs.

Methods: Eighteen patients who had arthroscopically confirmed partial MMPRTs were included. As a control, 18 patients who underwent partial meniscectomy for other types of MM tears were evaluated. Isolated partial MMPRTs were classified into the following three types: type A, accurate partial stable tear (cleavage $<1 / 2$ of root width); type B, bridged unstable root tear (cleavage $\geq 1 / 2$ of root width); type C, complex horn tear expanded to the root. Conventional MRI-based findings of MMPRTs were evaluated between two groups $(n=23)$. Posterior root irregularity, bone marrow spot, and ocarina-like appearance showing several condensed circles in triangular meniscal horn (ocarina sign) were also evaluated.
\end{abstract}

Results: Posterior root irregularity and bone marrow spot were frequently observed in the partial MMPRTs (47.8\%), compared with the other MM tears ( $P=0.007$ and 0.023 , respectively). The ocarina sign was detected in $69.6 \%$ of patients with partial MMPRTs. A significant difference between two groups was observed in a positive ratio of ocarina sign $(P<0.001)$. Types $A, B$, and $C$ of the partial tear/damage were observed in three, eight, and seven patients, respectively. The ocarina sign was the most common MRI finding in each type of partial MMPRT.

Conclusions: This study demonstrated that a characteristic MRI finding, "ocarina sign," was frequently observed in patients with partial tear/damage of the MM posterior root. The ocarina sign was the most common MRI finding in several types of partial MMPRTs. Our results suggest that the ocarina sign may be useful to diagnose unnoticed partial MMPRTs.

Level of evidence: IV, retrospective comparative study.

Keywords: Medial meniscus, Posterior root, Partial tear, Magnetic resonance imaging, Ocarina sign

*Correspondence: matino@md.okayama-u.ac.jp

Department of Orthopaedic Surgery, Okayama University Hospital, 2-5-1

Shikatacho, Kitaku, Okayama 700-8558, Japan

\section{Background}

Posterior root tears of the medial meniscus (MM), including partial, complete radial, and/or oblique tears adjacent to the posterior root attachment, lead to progressive cartilage loss, osteoarthritis, and subchondral insufficiency fracture of the knee joint by disrupting the

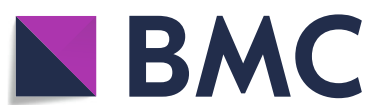

Part of Springer Nature

(c) The Author(s) 2021. Open Access This article is licensed under a Creative Commons Attribution 4.0 International License, which permits use, sharing, adaptation, distribution and reproduction in any medium or format, as long as you give appropriate credit to the original author(s) and the source, provide a link to the Creative Commons licence, and indicate if changes were made. The images or other third party material in this article are included in the article's Creative Commons licence, unless indicated otherwise in a credit line to the material. If material is not included in the article's Creative Commons licence and your intended use is not permitted by statutory regulation or exceeds the permitted use, you will need to obtain permission directly from the copyright holder. To view a copy of this licence, visit http://creativecommons.org/licenses/by/4.0/. The Creative Commons Public Domain Dedication waiver (http://creativeco mmons.org/publicdomain/zero/1.0/) applies to the data made available in this article, unless otherwise stated in a credit line to the data. 
MM functions [1]. With a greater understanding of posttraumatic knee degradation following MM posterior root tear (MMPRT), more emphasis has been placed on accurate diagnosis and early identification of MMPRT by magnetic resonance imaging (MRI) examinations $[2,3]$. Several characteristic MRI findings have been reported to detect MMPRT. In coronal images, giraffe neck sign and cleft/truncation sign (vertical linear defect) are useful to diagnose MMPRT [4-8]. In sagittal images, a ghost (or white meniscus) sign that shows a disappearance of the MM posterior root/horn on some slices can diagnose MMPRT with high sensitivity. A radial tear sign (radial linear defect) with complete discontinuity of the posterior root and fluid gap on axial images also has high diagnostic value in the diagnosis of MMPRT [4-6]. On the other hand, MM medial extrusion sign is often observed in the other types of MM tears and has a lower accuracy for detecting MMPRT compared with giraffe neck, cleft, ghost, and radial tear signs [5, 6]. However, these signs are clinically useful to detect complete tears of the MM posterior root. Diagnosing partial tear and/or pathological damage of the MM posterior root is still difficult.

A partial tear of the MM posterior root sometimes progresses to a complete radial root tear of the MM during nonoperative management [9]. Choi et al. report the MMPRTs as posterior MM root ligament lesions, and these lesions include three types of MRI-based appearances: degeneration, characterized by thickening of the root with intrasubstance hyperintensity not contacting the articular surface; partial tear, characterized by abnormal signal intensity extending to the articular surface or abnormal morphology of the root with partial root discontinuity; and complete tear, characterized by complete discontinuity of the affected root [10]. Palisch et al. describe that a partial radial tear of the MM posterior root (LaPrade arthroscopic/morphologic classification type 1 [11]) shows a fluid signal intensity at the root insertion and the subchondral and/or subenthesial linear bone marrow signal intensity on MRI [2]. However, the MM posterior root usually consists of multiple fiber bundles, typically exhibited as hyperintensity-/hypointensitymixed signals (most of which are stripe-like signals) on MRI [12]. Many other meniscus tears at the MM posterior horn (horizontal, radial, and/or complex tears) show subcortical cystic lesions and/or posterior shiny-corner lesions (bone marrow lesions at the meniscal-covered portions of the tibial plateau) around the MM posterior root insertion [13, 14]. In addition, these studies have not proven congruity between MRI-based findings and actual arthroscopic views of meniscus tears.

The aim of this study was to evaluate diagnostic values involved in conventional MRI features of the MMPRT and find other MRI-based findings in patients with arthroscopically confirmed partial tears of the MM posterior root. We hypothesized that specific degenerative findings at the MM posterior root/horn on MRI are clinically useful to identify partial tear/damage of the MM posterior root.

\section{Patients and methods}

This study received the approval of our institutional review board, and written informed consent was obtained from all patients. Eighteen patients who underwent transtibial pullout repairs for arthroscopically confirmed partial tears of the MM posterior root between February 2018 and December 2020 were included (Table 1). As a control group, 18 patients who underwent partial meniscectomy for other types of MM tears between July 2018 and November 2020 were evaluated (Table 1). All the patients required arthroscopic treatments for symptomatic knee pains and were diagnosed as having isolated MM tears by MRI examinations and arthroscopic findings [15]. Details of sudden posteromedial painful popping, a characteristic episode of patients with MMPRTs, were obtained from the patients by careful interviews $[16,17]$. Patient demographics are presented in Table 1.

\section{Arthroscopic evaluations of the MM tears}

Following the medial joint space-widening procedure (outside-in pie-crusting technique [18]), partial MMPRTs were determined by careful arthroscopic examinations according to the meniscal root tear classification [11]. A partial tear/damage of the MM posterior root was defined as an incomplete structural cleavage between 0 and $9 \mathrm{~mm}$ from the native MM posterior root attachment. An "accurate" partial stable tear (type A) of the MM posterior root was determined by careful

Table 1 Patient demographics

\begin{tabular}{lllr}
\hline & Partial MMPRTs & Other MM tears & $P$ value \\
\hline Number of patients & 18 & 18 & \\
Gender, men/women & $2 / 16$ & $11 / 7$ & $0.005^{* a}$ \\
Age (years) & $64.6 \pm 7.8$ & $54.0 \pm 9.0$ & $0.001^{*}$ \\
Height (m) & $1.54 \pm 0.06$ & $1.66 \pm 0.09$ & $<0.001^{*}$ \\
Body weight (kg) & $62.3 \pm 11.2$ & $69.4 \pm 13.5$ & $0.046^{*}$ \\
Body mass index (kg/ & $26.2 \pm 3.9$ & $25.1 \pm 3.7$ & 0.184 \\
m²) & & & $0.060^{\text {a }}$ \\
Painful popping episode & $8(44.4)$ & $2(11.1)$ & \\
(\%) & & & 23 \\
MRl examinations & 23 & & \\
\hline
\end{tabular}

Data of age, height, body weight, and body mass index are displayed as mean \pm standard deviation. MMPRT medial meniscus posterior root tear, MM medial meniscus. Statistical differences in age, height, body weight, and body mass index between two groups were analyzed using Mann-Whitney $U$ test. aFisher's exact test. *Significant difference 

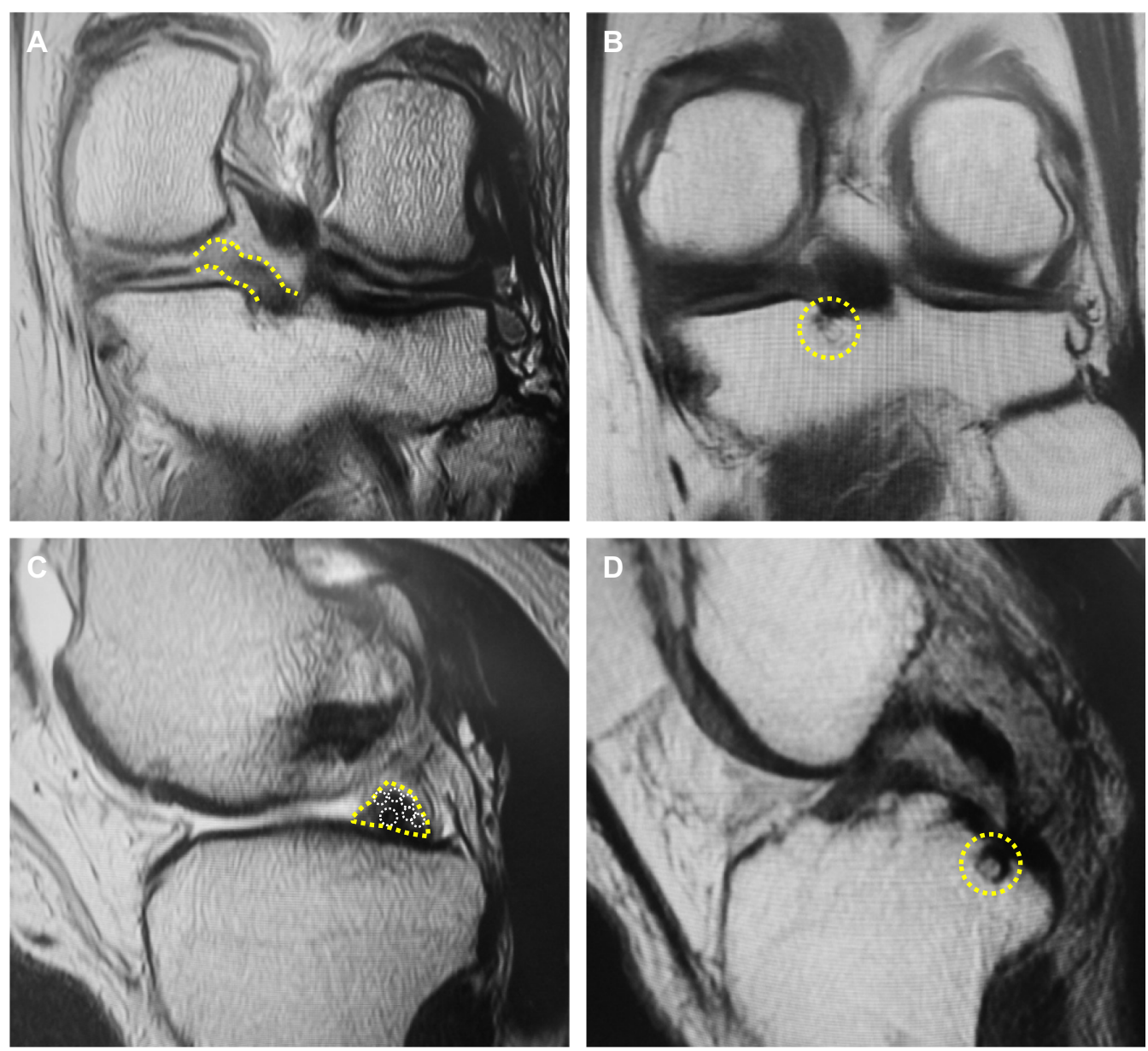

Fig. 1 Characteristic MRI finding in partial tear/damage of the MM posterior root. A A root irregularity (dotted area, coronal view). B A bone marrow spot (subcortical cyst) under the posterior root attachment (dotted circle, coronal view). C An ocarina sign (dotted area, sagittal view nearby the MM posterior root). D A bone marrow spot (dotted circle, sagittal view)

observation with probing. A structural cleavage with the root width of less than one half was classified as a type A partial tear. A superficially "bridged" root covered with connective tissues was classified as a type B partial tear that showed a structural cleavage with root width of one half or more. A "complex" tear of the MM posterior horn expanded to the posterior root was classified as a type $\mathrm{C}$ partial tear. A completely detached root with a gap was excluded in this study. Other types of MM tears were determined by arthroscopic findings and MRI scans: ten complex tears involved in degenerated horizontal/flap tears, four flap tears, three degenerated horizontal tears, and one degenerated bucket-handle tear.

A partial tear/damage of the MM posterior root was divided into the following three types: type $\mathrm{A}$, accurate partial stable tear (cleavage $<1 / 2$ of root width); type B, bridged unstable root tear (cleavage $\geq 1 / 2$ of root width); type $\mathrm{C}$, complex horn tear expanded to the root.

\section{Assessments of MRI scans}

Retrospective re-evaluations of preoperative MRIs were performed after the surgeries. Patients were examined by preoperative MRI scans (one to three times). The number of MRI examinations was 23 for each group (partial MMPRTs or other MM tears). MRI scans were mainly obtained using an Achieva 1.5T (Philips, Amsterdam, The Netherlands) or an EXCELART Vantage Powered by Atlas 1.5T (Toshiba Medical Systems, Otawara, Japan) with a knee coil. Standard sequences of the Achieva included sagittal [repetition time (TR)/echo time (TE) 742/18], coronal (TR/TE 637/18), and axial (TR/TE 499/18) T2-weighted fast-field echo with a $20^{\circ}$ flip angle (FA). Standard sequences of the Vantage included sagittal and coronal proton density (PD) fast-spin-echo (TR/TE 2300/18) and axial T2-weighted fat suppression (TR/TE $3500 / 60$ ) with a $90^{\circ} \mathrm{FA}$. Slice thickness was $3 \mathrm{~mm}$ with a $0.6 \mathrm{~mm}$ gap. Field of view (FOV) was 16 (or 17) $\mathrm{cm}$ with an acquisition matrix size of $205 \times 256$ (or $200 \times 368$ ) [6, 


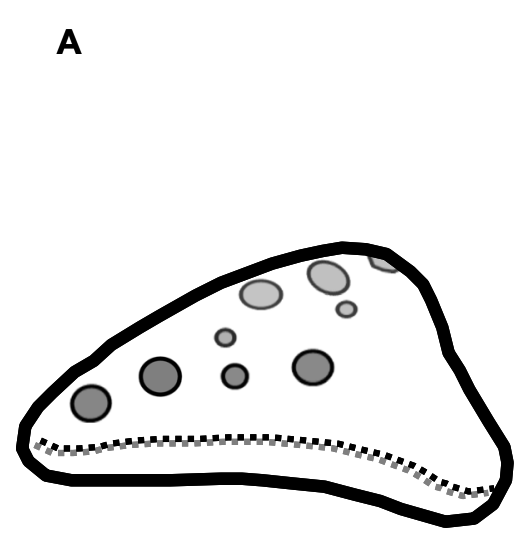

\section{Ocarina sign}

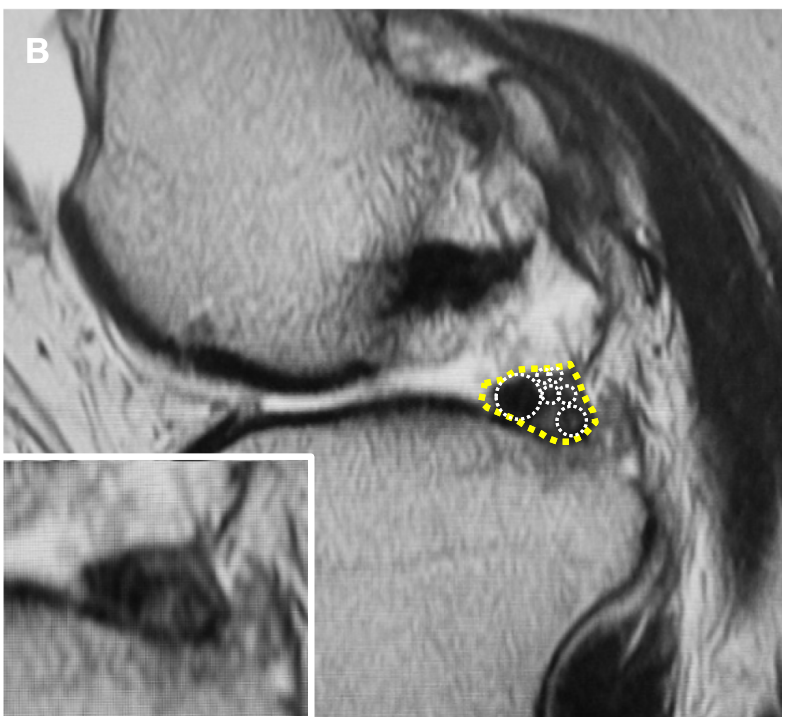

Fig. 2 An ocarina sign. A An illustration of an ocarina sign. B A sagittal image of a partial MMPRT. Note a multiple fiber bundle formation showing several condensed circles (white circles) in triangular meniscal horn (yellow dotted area), an ocarina-like appearance. An inlet shows the original image

Table 2 Characteristic MRI findings in partial/complete MMPRTs

\begin{tabular}{llll}
\hline & Partial MMPRTs & Other MM tears & $P$ value \\
\hline Coronal images & & & \\
Cleft sign (\%) & $4(17.4)$ & $0(0.0)$ & $0.109^{\mathrm{a}}$ \\
Giraffe neck sign (\%) & $5(21.8)$ & $0(0.0)$ & $0.049^{* a}$ \\
MM extrusion (mm) & $3.21 \pm 1.07$ & $2.84 \pm 1.21$ & $0.142^{\mathrm{b}}$ \\
MM extrusion > 3 mm & $13(56.5)$ & $12(52.2)$ & $1.000^{\mathrm{a}}$ \\
$\quad(\%)$ & & & \\
Root irregularity (\%) & $11(47.8)$ & $2(8.70)$ & $0.007^{* \mathrm{a}}$ \\
Bone marrow spot (\%) & $11(47.8)$ & $3(13.0)$ & $0.023^{* a}$ \\
Sagittal images & & & $1.000^{\mathrm{a}}$ \\
Ghost sign (\%) & $1(4.3)$ & $0(0.0)$ & $<0.001^{* a}$ \\
Ocarina sign (\%) & $16(69.6)$ & $3(13.0)$ & $0.071^{\mathrm{a}}$ \\
Bone marrow spot (\%) & $8(34.8)$ & $2(8.70)$ & $0.233^{\mathrm{a}}$ \\
Axial images & & & $0(0.0)$ \\
Radial tear sign (\%) & $3(13.0)$ &
\end{tabular}

Data of medial meniscus (MM) extrusion are displayed as mean \pm standard deviation. MRI magnetic resonance imaging, MMPRT medial meniscus posterior root tear. ${ }^{a}$ Fisher's exact test. ${ }^{b}$ Mann-Whitney $U$ test. ${ }^{*}$ Significant difference

19]. Coronal images were obtained, along with a section parallel to a tangential line between both posterior femoral condyles. Sagittal images were set perpendicular to the coronal images. Axial images were obtained according to the position of both menisci.

Conventional MRI-based findings of the MMPRT such as cleft, giraffe neck, medial extrusion, ghost, and radial tear signs [6] were evaluated. Medial extrusion of the MM was measured from the medial margin of the tibial plateau to the outer border of the MM on the coronal image that crossed the midpoint of the anteroposterior length of the MM. Medial extrusion $(>3 \mathrm{~mm}$ ) was defined as a positive extrusion sign.

During MRI evaluations, we noticed that root irregularity and bone marrow spot at the posterior root attachment were frequently observed in coronal/sagittal images of the patients (Fig. 1). A bone marrow spot (subcortical cyst) was defined as foci of markedly increased signal intensity in the subcortical bone with rounded and well-circumscribed margins and no evidence of internal marrow tissue or trabecular bone on both the T2 and PD-weighted images [13]. In sagittal images, a multiple fiber bundle formation showing several condensed circles in triangular meniscal horn, an ocarina-like appearance, was observed with considerable frequency (Figs. 1, 2). Root irregularity, bone marrow spot, and "ocarina sign" were evaluated as characteristic MRI findings to identify partial tear/damage of the MM posterior root.

\section{Statistical analysis}

Data were presented as means \pm standard deviations. Statistical differences in age, height, body weight, body mass index, and MM extrusion between two groups were analyzed using Mann-Whitney $U$-test. Differences in gender, positive ratio of painful popping episode, and MRI signs between groups were compared using Fisher's exact test. Power and statistical analyses were performed using EZR (Saitama Medical Center, Saitama, Japan), which is a graphical user interface for $\mathrm{R}$ (The $\mathrm{R}$ Foundation for 

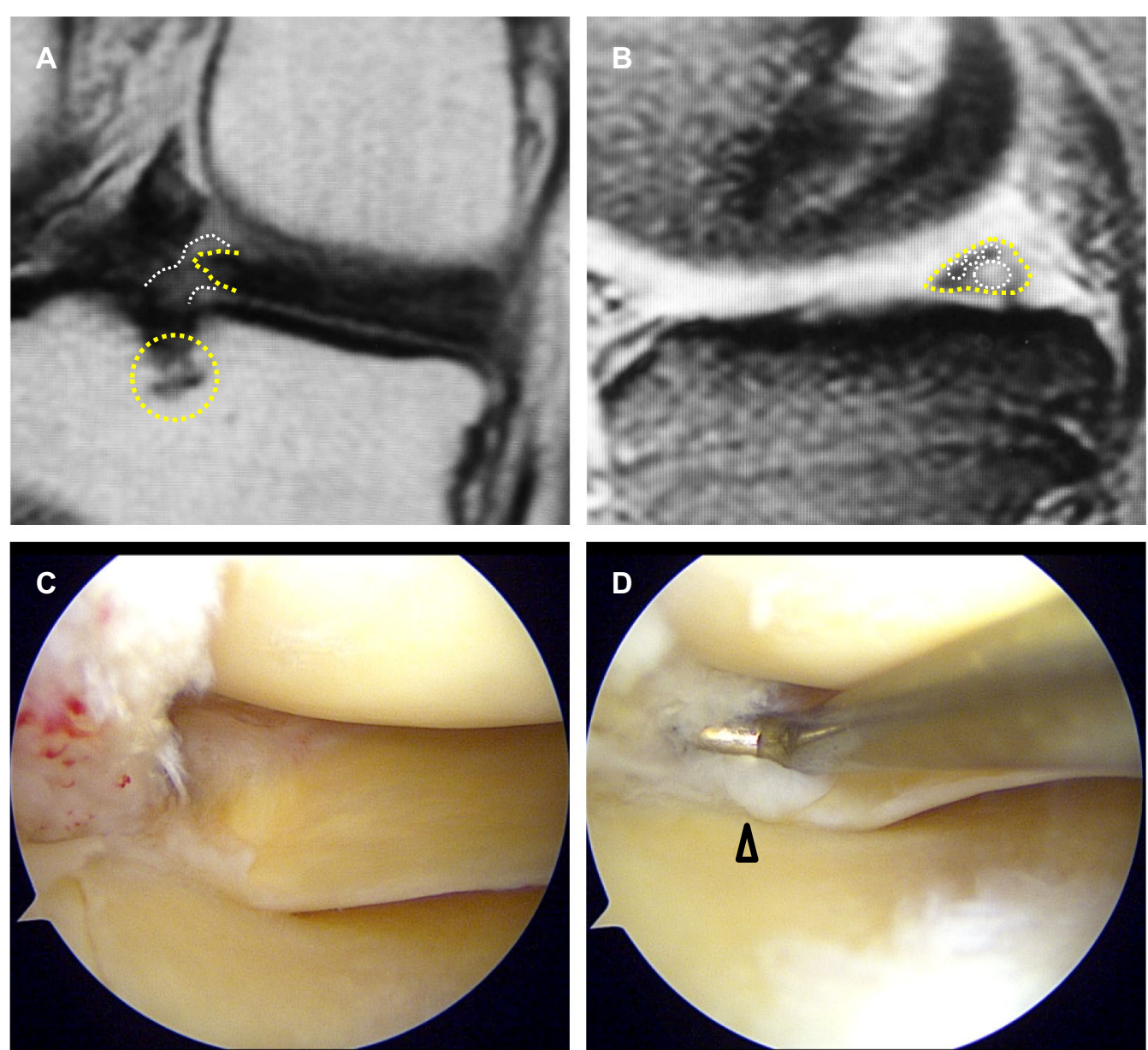

Fig. 3 An "accurate" partial stable tear (type A) of the MM posterior root (64-year-old woman). A A posterior root irregularity (dashed area, PD-weighted coronal view). Bone marrow spots (yellow circle). B An ocarina sign (T2* sagittal view). C, D Arthroscopic findings from the anterolateral portal. A structural cleavage with root width > 1/2 (arrowhead)

Statistical Computing). Significance was set to $P<0.05$. Two orthopedic surgeons independently assessed MRIs in a blinded manner. Each observer performed each evaluation twice, at least 2 weeks apart. The reliability of MRI evaluation was assessed by examining the interobserver and intraobserver reliabilities. The interobserver and intraobserver reliabilities were assessed using an intraclass correlation coefficient (ICC). ICC $>0.80$ was considered to represent a reliable measurement.

\section{Results}

The incidence of the MMPRT was higher in women compared with that of other MM tears $(P=0.005$, Table 1$)$. Significant differences between the MMPRT and other MM tear groups were observed in age $(P=0.001)$, height $(P<0.001)$, and body weight $(P=0.046)$. Posteromedial painful popping of the knee was not a significant characteristic episode in patients with partial MMPRTs (44.4\%) compared with other MM tears (11.1\%).
Conventional MRI findings of MMPRTs were evaluated in both groups (Table 2). A giraffe neck sign was observed in $21.8 \%$ of the MRIs with partial MMPRTs $(P=0.049)$. On the other hand, no significant differences between two groups were detected in cleft sign, MM extrusion, ghost sign, and radial tear sign (Table 2).

Posterior root irregularity and bone marrow spot (subcortical cyst) on coronal images were frequently observed in the partial MMPRTs (47.8\%), with significant differences compared with other MM tears $(P=0.007$ and 0.023 , respectively). In sagittal images, a multiple fiber bundle formation that showed an ocarina-like appearance in triangular MM posterior horn (ocarina sign) was observed on $69.6 \%$ of MRI scans of the partial MMPRT group (Table 2, Figs. 1, 2). A significant difference between two groups was detected in a positive ratio of ocarina sign $(P<0.001)$. No significant difference between the groups was observed in bone marrow spot sign on sagittal images $(P=0.071)$. 

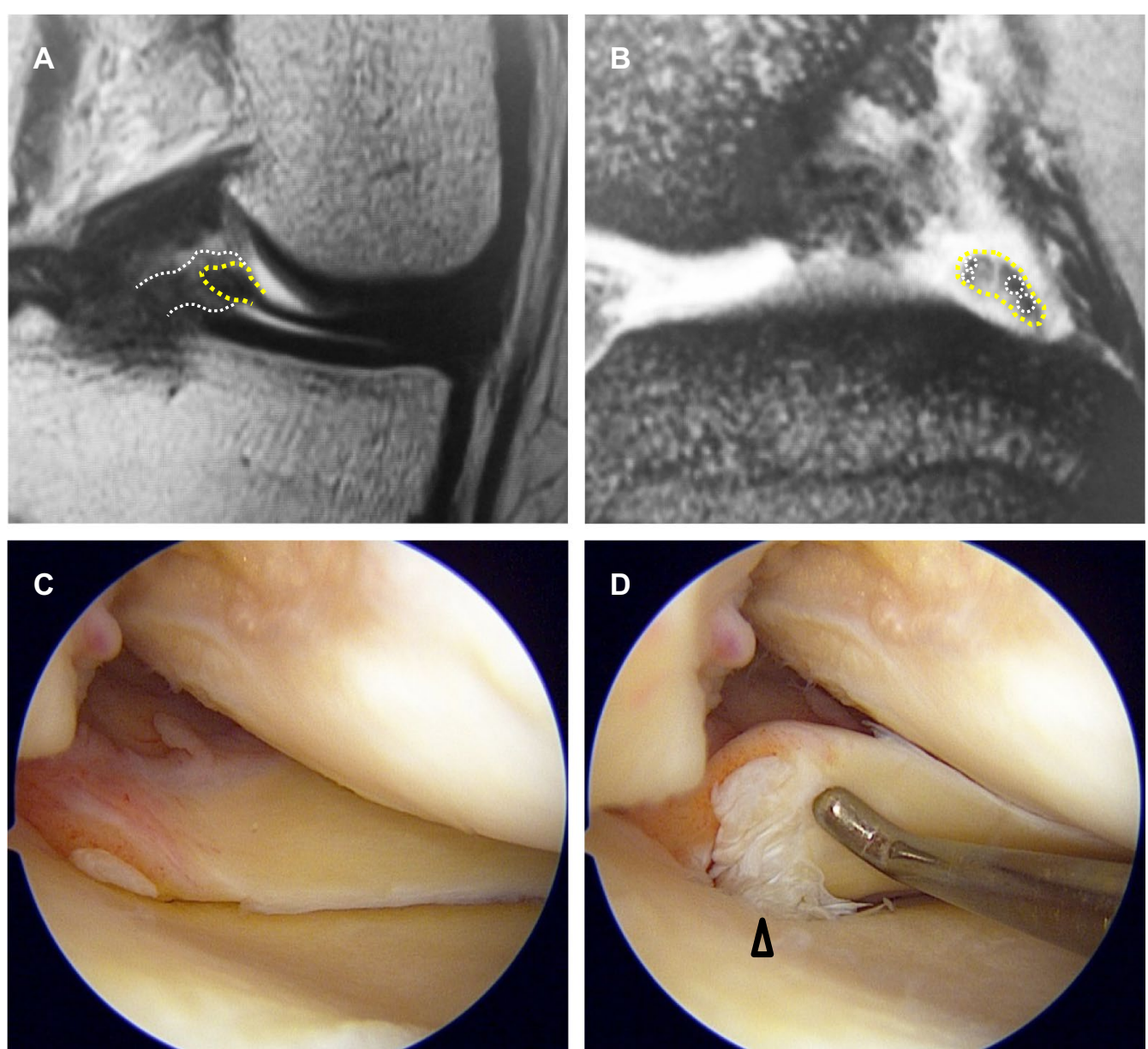

Fig. 4 A"bridged" unstable root tear (type B) of the MM posterior root (61-year-old man). A A posterior root irregularity (dashed area, coronal view). B An ocarina sign (T2* sagittal view). C, D Arthroscopic findings from the anterolateral portal. An unstable tear ( $\geq 1 / 2$ of root width) with connective tissue coverage (arrowhead)

Accurate partial stable tears (cleavage $<1 / 2$ of root width) of the MM posterior root were observed in three patients (type A, Fig. 3). Bridged unstable root tears (cleavage $\geq 1 / 2$ of root width, type B, Fig. 4) and complex posterior horn tears expanded to the root (type C, Fig. 5) were observed in eight and seven patients, respectively. These three types of partial MMPRTs showed some continuity between the posterior root and horn in both arthroscopic and MRI findings. Ocarina sign was the most common MRI finding in each type of partial MMPRT (Table 3). There were no individual features of ocarina sign in each type of partial MMPRT. The interobserver reproducibility and intraobserver repeatability of the MRI findings were satisfactory, with mean ICC values of 0.87 and 0.89 , respectiv ely.

\section{Discussion}

The most important finding in this study was that a new characteristic MRI finding, "ocarina sign," was frequently observed in patients with partial tear/damage of the MM posterior root. In addition, the ocarina sign was the most common MRI finding in several types of partial MMPRTs. Our results suggest that the ocarina sign may be a useful MRI finding to diagnose an unnoticed partial tear/damage of the MM posterior root.

Choi et al. describe that partial tears of the MM posterior root were observed in $11.7 \%$ of 419 symptomatic knee MRIs [10]. Degenerations and complete tears of the MM posterior root were detected in $14.3 \%$ and $2.6 \%$ of MRI scans in patients with symptomatic knees, respectively. In this literature, the rate of partial tears account 

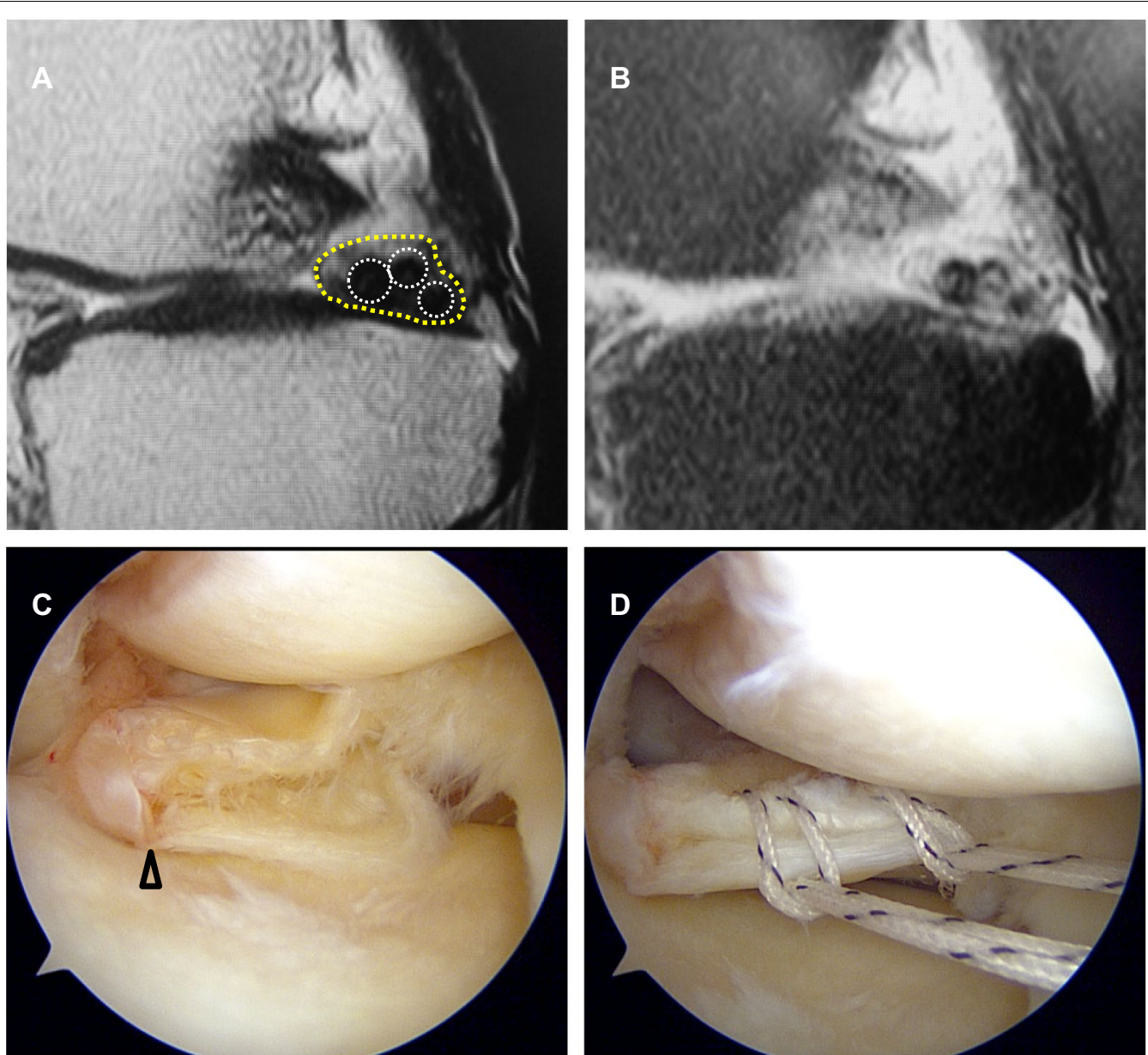

Fig. 5 A "complex" tear of the MM posterior horn expanded to the MM posterior root (type C, 58-year-old woman). A An ocarina sign (sagittal view). B PD fat-saturated sagittal view. C Arthroscopic findings from the anterolateral portal. A complex posterior horn tear expanded to the root (arrowhead). D Two cinch stitches for posterior root repair

Table 3 Characteristic MRI findings in each type of partial MMPRTS

\begin{tabular}{llll}
\hline & Type A $(\boldsymbol{n}=\mathbf{3})$ & Type B $(\boldsymbol{n}=\mathbf{8})$ & Type C $(\boldsymbol{n}=\mathbf{7})$ \\
\hline Coronal images & & & \\
Cleft sign & 0 & 3 & 0 \\
Giraffe neck sign & 0 & 3 & 1 \\
Root irregularity & 0 & 6 & 3 \\
Bone marrow spot & 3 & 3 & 3 \\
Sagittal images & & & \\
Ghost sign & 0 & 1 & 0 \\
Ocarina sign (\%) & $2(67)$ & $6(75)$ & $5(71)$ \\
Bone marrow spot & 2 & 3 & 2 \\
Axial images & & 2 & 1 \\
Radial tear sign & 0 & 2 & \\
\hline
\end{tabular}

Evaluations of most recent magnetic resonance imaging (MRI) before surgery. MMPRT medial meniscus posterior root tear for 40.8\% (49/120) of MRI-based posterior MM root ligament lesions, despite a lower rate of complete tears $(9.2 \%$, 11/120) [10]. On the other hand, Moon et al. describe that three $(4.8 \%)$ patients showed partial MMPRTs corresponding to LaPrade classification type 1 tears in arthroscopic image analyses of 63 patients [20]. They report that complete radial (type 2) and complex oblique (type 4) tears accounted for $88.9 \%$ and $6.3 \%$ of the MMPRTs, respectively [20]. Several authors demonstrate that the rate of type 1 partial MMPRTs accounts for 3.9\% (5/128 knees) [21], 7.2\% (6/83 knees) [22], and 16.4\% (19/116 knees) [23] in patients with MMPRTs who underwent arthroscopic treatments. Based on these findings, most of the patients diagnosed with MRI-based partial MMPRTs may not require immediate surgical treatments. Many of the partial tear/damage of the MM posterior root may not be noticed on clinical examination and MRI assessment, even if a surgeon request MRI scans. We consider that several useful MRI findings such as ocarina sign, 
root irregularity, and bone marrow spot can help surgeons and radiologists to make a reliable diagnosis of partial MMPRTs for preventing further progression to complete MMPRTs.

This study demonstrated that the incidence of the MMPRT was higher in women compared with that of other types of MM tears (Table 1). In addition, the MMPRTs were detected in older patients (mean age, $64.6 \pm 7.8$ years) compared with other MM tears (54.0 \pm 9.0 years). Several authors report that MMPRTs can occur particularly in middle-aged or older female patients who have a sudden posteromedial painful popping sensation of the knee during light activities such as using stairs and walking [5, 16, 17]. The posteromedial painful popping sensation is a useful clinical symptom to identify MMPRTs, and its sensitivity is $35-87 \%$ in patients with MMPRTs [16, 24]. However, in our study, no significant difference between partial MMPRT and other MM tear groups was observed in the positive ratio of painful popping episode (Table 1). A relatively low rate $(44 \%)$ of the painful popping episode may be caused by the presence of type $\mathrm{C}$ complex posterior horn tear (Fig. 5) that expands to the MM posterior root gradually. On the other hand, five out of eight patients (63\%) who had type B bridged unstable root tears, similar to complete radial MMPRTs, remembered the popping episode.

In human cadaveric knees, fibrocartilage metaplasia and calcification increase in the MM posterior roots is associated with the degree of the MMPRT (partial or complete tears). A decrease of type I collagen deposition and an increase of type II collagen synthesis is observed in the extracellular matrix of the MM posterior root in osteoarthritic knees [25]. In addition, meniscus cell clusters that show depositions of type II collagen, aggrecan, and safranin $\mathrm{O}$-stained proteoglycans are frequently observed around meniscus tears [26]. These findings suggest that age-dependent degenerative changes in the MM posterior root may lead to partial and/or complete MMPRTs in older patients. We consider that the ocarina sign may represent some fiber-bundle disorganization and fibrocartilage formation around the junction between the MM posterior root and horn. Further investigations using histological analyses will be required to understand the ocarina-like appearance in MRI scans. An anatomic attachment of the MM posterior root is surrounded with the lateral edge of the medial tibial plateau, the anterior border of the posterior cruciate ligament, and the retro-eminence ridge. The maximum anteroposterior length of the MM posterior root attachment is a mean of $7.7 \mathrm{~mm}$ at the sagittal plane passing through the peak of the medial intercondylar tubercle [27]. The mediolateral width of the MM posterior root attachment is shorter than its anteroposterior length [27, 28]. In our study, the difference in the detection rate of bone marrow spot under the MM posterior root attachment was observed between coronal and sagittal images on MRI scans (Table 2). This may be caused by the difference in the number of MRI sections passing through the anatomic attachment of the MM posterior root.

There are several limitations in this study. Our study was a retrospective comparative study that included a small number of patients. MRI examinations were performed differently (number of examination times, variable protocols, and duration from injury to MRI scans). There is a possibility that the appearance of the ocarina sign may depend on the duration between injury and MRI examination. We evaluated several characteristic MRI findings in a single knee flexion angle $\left(10^{\circ}\right)$ under non-weight-bearing condition. MRI assessments of the MM posterior root attachment using thin slices and appropriate planes under loading condition may enhance the diagnostic value of these characteristic MRI findings in identifying partial tear/damage of the MM posterior root.

\section{Conclusions}

This study demonstrated that a characteristic MRI finding, the "ocarina sign," was frequently observed in patients with partial tear/damage of the MM posterior root. In addition, the ocarina sign was the most common MRI finding in several types of partial MMPRTs. Our results suggest that the ocarina sign, root irregularity, and bone marrow spot under the root attachment may be useful MRI findings to diagnose unnoticed partial tears/damage of the MM posterior root.

\section{Abbreviations}

MM: Medial meniscus; MMPRT: Medial meniscus posterior root tear; MRI: Magnetic resonance imaging.

\section{Acknowledgements}

We thank Drs. Yoshiki Okazaki and Shinichi Miyazawa for their clinical supports.

\section{Authors' contributions}

TF designed the study, performed surgeries, and prepared the manuscript. $\mathrm{TH}$, $\mathrm{KK}, \mathrm{YO}, \mathrm{NH}, \mathrm{MT}$, and TO contributed to the data collection. All authors contributed to the analysis and interpretation of data. All authors critically reviewed the manuscript and approved the final version of the manuscript. All authors read and approved the final manuscript.

\section{Funding}

This study was partly supported by a grant from Japan Society for the Promotion of Science (21K09279, TF).

Availability of data and materials

Not applicable. 


\section{Declarations}

\section{Ethics approval and consent to participate}

This study received the approval of our institutional review board (Okayama University, \#1857), and written informed consent was obtained from all patients. All participants provided informed consent to participate in this study.

\section{Consent for publication}

All participants provided informed consent for the publication of this study.

\section{Competing interests}

The authors declare that they have no competing interests.

Received: 5 July 2021 Accepted: 23 September 2021

Published online: 09 October 2021

\section{References}

1. Bhatia S, LaPrade CM, Ellman MB, LaPrade RF (2014) Meniscal root tears: significance, diagnosis, and treatment. Am J Sports Med 42(12):3016-3030

2. Palisch AR, Winters RR, Willis MH, Bray CD, Shybut TB (2016) Posterior root meniscal tears: preoperative, intraoperative, and postoperative imaging for transtibial pullout repair. Radiographics 36(6):1792-1806

3. Kim DH, Lee GC, Kim HH, Cha DH (2020) Correlation between meniscal extrusion and symptom duration, alignment, and arthritic changes in medial meniscus posterior root tear: research article. Knee Surg Relat Res 32(1):2

4. Lee YG, Shim JC, Choi YS, Kim JG, Lee GJ, Kim HK (2008) Magnetic resonance imaging findings of surgically proven medial meniscus root tear: tear configuration and associated knee abnormalities. J Comput Assist Tomogr 32(3):452-457

5. Choi SH, Bae S, Ji SK, Chang MJ (2012) The MRI findings of meniscal root tear of the medial meniscus: emphasis on coronal, sagittal and axial images. Knee Surg Sports Traumatol Arthrosc 20(10):2098-2103

6. Furumatsu T, Fujii M, Kodama Y, Ozaki T (2017) A giraffe neck sign of the medial meniscus: a characteristic finding of the medial meniscus posterior root tear on magnetic resonance imaging. J Orthop Sci 22(4):731-736

7. Okazaki Y, Furumatsu T, Shimamura Y, Saiga K, Ohashi H, Uchino T, Kamatsuki Y, Okazaki Y, Ozaki T (2019) Time-dependent increase in medial meniscus extrusion after medial meniscus posterior root tear analyzed by using magnetic resonance imaging. Knee Surg Relat Res 31(2):120-125

8. Kodama Y, Furumatsu T, Kamatsuki Y, Hiranaka T, Takahata T, Sadakane M, Ikuta H, Yasumitsu M, Ozaki T (2019) Preliminary diagnosis of medial meniscus posterior root tears using the Rosenberg radiographic view. Knee Surg Relat Res 31(1):9

9. Krych AJ, LaPrade MD, Hevesi M, Rhodes NG, Johnson AC, Camp CL, Stuart MJ (2020) Investigating the chronology of meniscus root tears: do medial meniscus posterior root tears cause extrusion or the other way around? Orthop J Sports Med 8(11):2325967120961368

10. Choi JY, Chang EY, Cunha GM, Tafur M, Statum S, Chung CB (2014) Posterior medial meniscus root ligament lesions: MRI classification and associated findings. AJR Am J Roentgenol 203(6):1286-1292

11. LaPrade CM, James EW, Cram TR, Feagin JA, Engebretsen L, LaPrade RF (2015) Meniscal root tears: a classification system based on tear morphology. Am J Sports Med 43(2):363-369

12. Wang $P$, Zhang CZ, Zhang D, Liu QY, Zhong XF, Yin ZJ, Wang B (2018) The imaging features of the meniscal roots on isotropic 3D MRI in young asymptomatic volunteers. Medicine (Baltimore) 97(18):e0624

13. Son JY, Yoon YC, Jin W, Cha JG (2018) The prevalence and characteristics of a subcortical cystic lesion at the subspinous region of the knee. Acta Radiol 59(1):97-104
14. Chang EY, Chen KC, Chung CB (2014) The shiny corner of the knee: a sign of meniscal osteochondral unit dysfunction. Skeletal Radiol 43(10):1403-1409

15. Furumatsu T, Okazaki Y, Kodama Y, Okazaki Y, Kamatsuki Y, Masuda S, Hiranaka T, Ozaki T (2019) The accuracy of a newly developed guide system in medial meniscus posterior root repair: a comparison between two aiming guides. Knee Surg Relat Res 31(1):7

16. Bae JH, Paik NH, Park GW, Yoon JR, Chae DJ, Kwon JH, Kim JI, Nha KW (2013) Predictive value of painful popping for a posterior root tear of the medial meniscus in middle-aged to older Asian patients. Arthroscopy 29(3):545-549

17. Furumatsu T, Okazaki Y, Okazaki Y, Hino T, Kamatsuki Y, Masuda S, Miyazawa S, Nakata E, Hasei J, Kunisada T, Ozaki T (2019) Injury patterns of medial meniscus posterior root tears. Orthop Traumatol Surg Res 105(1):107-111

18. Todor A, Caterev S, Nistor DV (2016) Outside-in deep medial collateral ligament release during arthroscopic medial meniscus surgery. Arthrosc Tech 5(4):e781-e785

19. Furumatsu T, Miyazawa S, Tanaka T, Okada Y, Fujii M, Ozaki T (2014) Postoperative change in medial meniscal length in concurrent all-inside meniscus repair with anterior cruciate ligament reconstruction. Int Orthop 38(7):1393-1399

20. Moon HS, Choi CH, Jung M, Lee DY, Hong SP, Kim SH (2021) Early surgical repair of medial meniscus posterior root tear minimizes the progression of meniscal extrusion: response. Am J Sports Med 49(1):NP3-5

21. Kamatsuki Y, Furumatsu T, Hiranaka T, Okazaki Y, Kodama Y, Kintaka K, Ozaki T (2021) Accurate placement of a tibial tunnel significantly improves meniscal healing and clinical outcomes at 1 year after medial meniscus posterior root repair. Knee Surg Sports Traumatol Arthrosc. https://doi.org/10.1007/s00167-020-06376-9

22. Furumatsu T, Hiranaka T, Okazaki Y, Kintaka K, Kodama Y, Kamatsuki Y, Ozaki T (2021) Medial meniscus posterior root repairs: a comparison among three surgical techniques in short-term clinical outcomes and arthroscopic meniscal healing scores. J Orthop Sci S09492658(20):30353-30355. https://doi.org/10.1016/j.jos.2020.11.013

23. Kim JY, Bin SI, Kim JM, Lee BS, Oh SM, Cho WJ (2019) A novel arthroscopic classification of degenerative medial meniscus posterior root tears based on the tear gap. Orthop J Sports Med 7(3):2325967119827945. https:// doi.org/10.1177/2325967119827945

24. Furumatsu T, Kamatsuki Y, Fujii M, Kodama Y, Okazaki Y, Masuda S, Ozaki T (2017) Medial meniscus extrusion correlates with disease duration of the sudden symptomatic medial meniscus posterior root tear. Orthop Traumatol Surg Res 103(8):1179-1182

25. Park DY, Min BH, Choi BH, Kim YJ, Kim M, Suh-Kim H, Kim JH (2015) The degeneration of meniscus roots is accompanied by fibrocartilage formation, which may precede meniscus root tears in osteoarthritic knees. Am I Sports Med 43(12):3034-3044

26. Kodama Y, Furumatsu T, Maehara A, Ozaki T (2018) Composition of cell clusters in torn menisci and their extracellular matrix components. Acta Med Okayama 72(5):499-506

27. Hino T, Furumatsu T, Miyazawa S, Fujii M, Kodama Y, Kamatsuki Y, Okazaki Y, Masuda S, Okazaki Y, Ozaki T (2020) A histological study of the medial meniscus posterior root tibial insertion. Connect Tissue Res 61(6):546-553

28. Brody JM, Hulstyn MJ, Fleming BC, Tung GA (2007) The meniscal roots: gross anatomic correlation with 3-T MRI findings. AJR Am J Roentgenol 188(5):W446-W450

\section{Publisher's Note}

Springer Nature remains neutral with regard to jurisdictional claims in published maps and institutional affiliations. 